used as a precision measure. The model was internally validated with bootstrap samples to correct overfitting.

Results: The median follow-up times was 19.4 months (range, 0.77-106.13 months), and the 3-year OS was 41.8\% ( $95 \%$ confidence interval $[\mathrm{CI}]=29.9 \%-58.3 \%$ ). We found International Federation of Gynecology and Obstetrics (FIGO) stage, and adjuvant chemotherapy were independent factors for OS. The $\mathrm{CP}$ of the nomogram integrating with body mass index (BMI), FIGO stage and adjuvant chemotherapy was 0.72 $(95 \% \mathrm{CI}=0.70-0.75)$. The calibration curves for probability of 3-year OS also demonstrated agreement between nomogram prediction and observation data.

Conclusion: The nomogram to predict 3-year OS of UCSs after surgery with simple parameters include BMI, FIGO stage, and adjuvant chemotherapy was implemented in a nomogram and provides accurate prediction of individual patients' prognosis useful for patient counselling and deciding on follow-up strategies.

Poster (E21)

Endometrial Hyperplasia, Endometrial Intra-epithelial Neoplasia, and Endometrial Cancer

https://doi.org/10.3802/jgo.2021.32.S1.E21

\section{A validation study of a modified TCGA classification for patients with endometrial cancer treated with radical surgery and adjuvant chemotherapy}

\footnotetext{
Hiroyuki Yamazaki, ${ }^{1,}$ Kanako Hatanaka, ${ }^{2}$ Hiroshi Asano, Matsuno Yoshihiro, ${ }^{3}$ Yutaka Hatanaka, ${ }^{2}$ Hidemichi Watari

'Department of Obstetrics and Gynecology, Graduate School of Medicine and Faculty of Medicine, Hokkaido University, Sapporo, Japan (cvm5396@elms.hokudai.ac.jp)

${ }^{2}$ Center for Development of Advanced Diagnostics, Research Division of Genome Companion Diagnostics, Hokkaido University Hospital, Sapporo, Japan

${ }^{3}$ Department of Surgical Pathology, Hokkaido University Hospital, Sapporo, Japan
}

Objective: The Cancer Genome Atlas (TCGA) molecular classification for endometrial cancer is expected to propose the most appropriate treatment strategy for each patient. However, the prognostic stratifications remain unclear when adjuvant chemotherapy is applied. This study aimed to validate a modified TCGA classification, ProMisE, for patients who underwent adjuvant chemotherapy at intermediate or high risk of recurrence.

Methods: From 2003 to 2015, the patients who underwent systematic lymphadenectomy were enrolled. The patients were classified by ProMisE using immunohistochemistry for mismatch repair-related molecules (MLH1, MSH2, MSH6, PMS2) and p53, and direct sequencing for hotspot mutations in POLE (exon 9, 13, and 14). The 5-year disease-specific survival rates (5y-DSS) were estimated by the Kaplan-Meier method. Results: A total of 182 patients were analyzed. The median age and follow-up period were 57.4-year-old and 105 months. The patients categorized to stage I, II, III, and IV were 97, 23, 51, and 11, respectively. One hundred twenty-two patients were diagnosed as endometrioid carcinoma (grade 1-2), and 60 were high-grade types, including endometrioid (grade 3), serous, and clear cell carcinomas. Based on ProMisE, 57 patients were categorized into mismatch-repair deficiency (MMR-d), 45 into POLE-EDM (exonuclease domain mutations), 15 into p53 wildtype, and 4 into p53 abnormal. The 5y-DSS was $92.9 \%, 100 \%$, $91.1 \%$, and $75.0 \%$, respectively.

Conclusion: We conclude that ProMisE can stratify the prognosis even when adjuvant chemotherapy was applied for intermediate- and high-risk for recurrence. The 5y-DSS of MMR-d patients seemed better than previous reports, partly because adjuvant chemotherapy could improve their prognosis.

Poster (E22)

Endometrial Hyperplasia, Endometrial Intra-epithelial Neoplasia, and Endometrial Cancer https://doi.org/10.3802/jgo.2021.32.S1.E22

\section{Association between high body mass index and endometrial pathology in premenopausal women with abnormal uterine bleeding}

\author{
Ruangsak Lertkhachonsuk," Nuttida Mahakit, \\ Woranit Apikulprapa, Patou Tantbairojn \\ Chulalongkorn University, Bangkok, Thailand (ruangsak@chula.md)
}

Objective: To find out the correlation between body mass index and endometrial pathology in premenopausal women with abnormal uterine bleeding.

Methods: A cross-sectional study was conducted in King Chulalongkorn Memorial Hospital during 1 January to 31 December 2019. All cases of abnormal uterine bleeding in premenopausal women who had endometrial pathology were recruited. All endometrial histopathology had been reviewed. Patient clinical profiles were recorded. Body mass index (BMI) was calculated by using weight in kilogram divided by square of height in meters. Then all cases were categorized by Asia Pacific BMI classification into underweight $\left(<18.5 \mathrm{~kg} / \mathrm{m}^{2}\right)$, normal weight (18.5-22.9 kg/m²), overweight (23-24.9 kg/m²) and obese $\left(>25 \mathrm{~kg} / \mathrm{m}^{2}\right)$. The pathological reports were classified into 3 groups: normal epithelium, endometrial hyperplasia, and endometrium cancer. Statistical analysis was done SPSS version 
21.0. The p-value of 0.05 was consider statistically significant. Results: There were 562 cases recruited in the study. Mean age of patients was 43 years. The mean BMI was $25.74 \mathrm{~kg} / \mathrm{m}^{2}$ (range, 14.38-66.12). The BMI was divided into 3 groups: 1 ) Normal weight (BMI, 18.5-22.9 kg/m²) was 210 cases (37.3\%); 2) Over-weight (BMI, 23-24.9 kg/m²) was 90 cases (16.0\%); and 3) Obese (BMI $\geq 25 \mathrm{~kg} / \mathrm{m}^{2}$ ) was 262 cases $(46.6 \%)$. The pathological reports demonstrated 36 cases of endometrial hyperplasia and 22 cases of endometrial cancer. There was a correlation between obese women and endometrial hyperplasia with odds ratio 5.9 and p-value 0.015 . However, there were no statistically significant between other BMI patterns and endometrial pathology.

Conclusion: There was a significant correlation between obesity and endometrial hyperplasia in premenopausal women with abnormal uterine bleeding.

Poster (E23)

Endometrial Hyperplasia, Endometrial Intra-epithelial Neoplasia, and Endometrial Cancer

https://doi.org/10.3802/jgo.2021.32.S1.E23

\section{Incidence of parametrial spreading in endometrial cancer at stages I and II in Siriraj Hospital}

\section{Suwanit Therasakvichya, "Sasimas Rujirawong, Boonlert Viriyapak, Atthapon Jaishuen}

Faculty of Medicine, Siriraj Hospital, Mahidol University, Bangkok, Thailand (suwanit.the@mahidol.ac.th)

Objective: To identify the incidence of parametrial involvement in endometrial cancer stages I and II and the related risk factors. Methods: The retrospective descriptive study was performed to review data from patients with endometrial cancer, clinical stages I and II who underwent surgical treatment as primary therapy. The data included patients' status, pathological reports (especially parametrial involvement), stage, adjuvant treatment, complications of treatment, and response of disease.

Results: The medical charts of 76 patients were reviewed and 3 cases were excluded from this study because of incomplete pathological report. The mean age was 58 years old. Most of them were early stage (IA, IB and II that defined according to International Federation of Gynecology and Obstetrics 2009 surgical stage with radical or modified radical hysterectomy), 31 (42.5\%) tumor grade I, and $76.7 \%$ endometrioid adenocarcinoma. Three patients (4.1\%) had parametrial involvement. The tumor spread into parametrium by lymphovascular space invasion in 2 cases and one case had lymph node metastasis. Because of the small number of cases, it is hard to use this data to identify the risk factors and correlation of parametrial involvement.

Conclusion: The incidence of parametrial involvement was too small. The benefit of radical or modified radical hysterectomy to identify parametrial involvement in endometrial cancer is not strongly suggested from the results of this study. A prospective study with adequate sample size and long term follow up should be considered.

Poster (E24)

Endometrial Hyperplasia, Endometrial Intra-epithelial Neoplasia, and Endometrial Cancer

https://doi.org/10.3802/jgo.2021.32.S1.E24

\section{Management of inoperable endometrial cancer}

\section{Supakorn Pitakkarnkul,' Saranya Chanpanitkitchot, ${ }^{2}$ Siriwan Tangjitgamol ${ }^{3, *}$}

'Gynecologic Oncology Division, National Cancer Institute, Bangkok, Thailand

${ }^{2}$ Department of Obstetrics and Gynecology, Rajavithi Hospital, College of Medicine, Rangsit University, Bangkok, Thailand ${ }^{3}$ Department of Obstetrics and Gynecology, Faculty of Medicine Vajira Hospital, Navamindradhiraj University, Bangkok, Thailand (siriwanonco@ yahoo.com)

Objective: Most existing information about treatment for inoperable epithelial-myoepithelial carcinoma (EMC) are derived from retrospective studies or case series. Without evidence-based data from randomized study, collective data from available reports would be helpful for a gynecologic oncologist to select an alternative treatment option for EMC patients who are not candidates for primary surgery.

Methods: This work collected and summarized data from literature review of relevant studies according to the treatment intention (definitive or neoadjuvant). Treatment given with a palliative aim, either chemo- or hormonal therapy, or best supportive care is beyond the scope of this review.

Results: The 3 major types of treatment for inoperable EMC were radiation therapy, chemotherapy, or their combination, and interval surgery. Radiation therapy alone (of different modes) was used as a sole definitive modality of therapy particularly for early-stage diseases which limited to uterine body, cervix, with or without parametrial invasion. A more common treatment modality was neoadjuvant treatment prior to surgery. Post-operative adjuvant treatment was also sometimes used depending mainly on the sites of disease and results of surgery. Data of neoadjuvant hormonal or radiation therapy were limited focusing on laboratory outcomes or having only small number of patients, respectively. Most neoadjuvant treatment before surgery was chemotherapy and 